\title{
The relation between patient discomfort and uncompensated forces of a patient support device for breast and regional lymph node radiotherapy
}

\author{
Bert Boute $^{\mathrm{a}, \mathrm{b}, *}$, Liv Veldeman ${ }^{\mathrm{a}, \mathrm{c}}$, Bruno Speleers ${ }^{\mathrm{a}}$, Annick Van Greveling ${ }^{\mathrm{c}}$, Tom Van Hoof ${ }^{\mathrm{d}}$, \\ Joris Van de Velde ${ }^{\mathrm{d}}$, Tom Vercauteren ${ }^{\mathrm{a}, \mathrm{c}}$, Wilfried De Neve ${ }^{\mathrm{a}, \mathrm{c}}$, Jan Detand ${ }^{\mathrm{b}}$

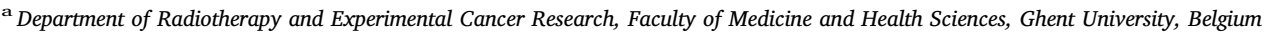 \\ ${ }^{\mathrm{b}}$ Industrial Design Centre, Faculty of Engineering and Architecture, Ghent University, Belgium \\ ${ }^{\mathrm{c}}$ Department of Radiation Oncology, University Hospital Ghent, Belgium \\ d Department of Anatomy, Faculty of Medicine and Health Sciences, Ghent University, Belgium
}

\section{A R T I C L E I N F O}

\section{Keywords:}

Comfort evaluation

Free-body diagram

Prone breast radiotherapy

\begin{abstract}
A B S T R A C T
Although many authors stated that a user-centred design approach in medical device development has added values, the most common research approach within healthcare is evidence-based medicine, which tend to focus on functional data rather than patient wellbeing and comfort. End user comfort is well addressed in literature for commercial products such as seats and hand tools but no data was found for medical devices.

A commercial patient support device for breast radiotherapy was analysed and a relation was found between discomfort and uncompensated internal body forces. Derived from CT-images, simplified patient free-body diagrams were analysed and pain and comfort evaluated. Subsequently, a new patient position was established and prototypes were developed. Patient comfort- and prototype optimization was done through iterative prototyping. With this approach, we were able to compensate all internal body forces and establish a force neutral patient free-body diagram. This resulted in comfortable patient positioning and favourable medical results.
\end{abstract}

\section{Introduction}

Currently, the two major concerns in medical device development (MDD) are improvement of patient safety and medical performance (Scanlon et al., 2006; Balka et al., 2006; Peters and Peters, 2007; Mullaney et al., 2012). Medical device tests are usually focused on functional data and conducted under technical vision and not in the real environment in which the devices will be used. However, non-functional data such as user emotions and usability aspects have not yet been fully covered (Tanure et al., 2015).

One of the most common research approaches within healthcare in the western world is evidence-based medicine. It has been defined as an approach to healthcare that "integrates individual clinical expertise with the best available external clinical evidence from systematic research in order to ensure the best prediction of outcomes in medical treatment" (Sackett, 1997; Sackett et al., 1996). The strong emphasis on clinical research in evidence-based medicine results in high level of importance upon functional data and measurable variables. Subsequently, they tend to distance themselves more from less quantitative (and functional) measurable aspects (such as patient wellbeing and comfort) (Mullaney et al., 2012). Healthcare professionals and administrators are recognizing the importance of a user centred care approach and that medical care delivered solely from a biomedical perspective is unable to produce an acceptable level of care, from a patient perspective (Mullaney et al., 2012; Edvardsson et al., 2006, 2008).

\subsection{Comfort}

End user comfort is well addressed in ergonomics literature for commercial products. One of the most researched is sitting comfort (De Looze et al., 2003). Little published work exists on end user comfort of medical devices (Martin et al., 2010; Sawyer et al., 1996). If research was conducted, topics such as patient safety and ergonomics (Martin et al., 2010; Clarkson et al., 2004), user comfort for medical staff (instruments) (Loring and Lemieux, 2010; Xiao, 2014), patient comfort during operations (Jensen et al., 2002) and medicine handling and dispensing (Dong and Vanns, 2009) were broached.

There are several factors which influence discomfort. As Zhang et al. (Cordell et al., 1995) conclude for sitting, physical factors such as ache, blood circulation cut-off, cramped and sore muscles, stiffness, etc. underlie discomfort. Additionally, shape elements of the device can also

\footnotetext{
* Corresponding author. Ghent University, Industrial Design Centre, faculty of Engineering and Architecture, Graaf Karel de Goedelaan 5, 8500 Kortrijk, Belgium.

E-mail address: bert.boute@ugent.be (B. Boute).
} 

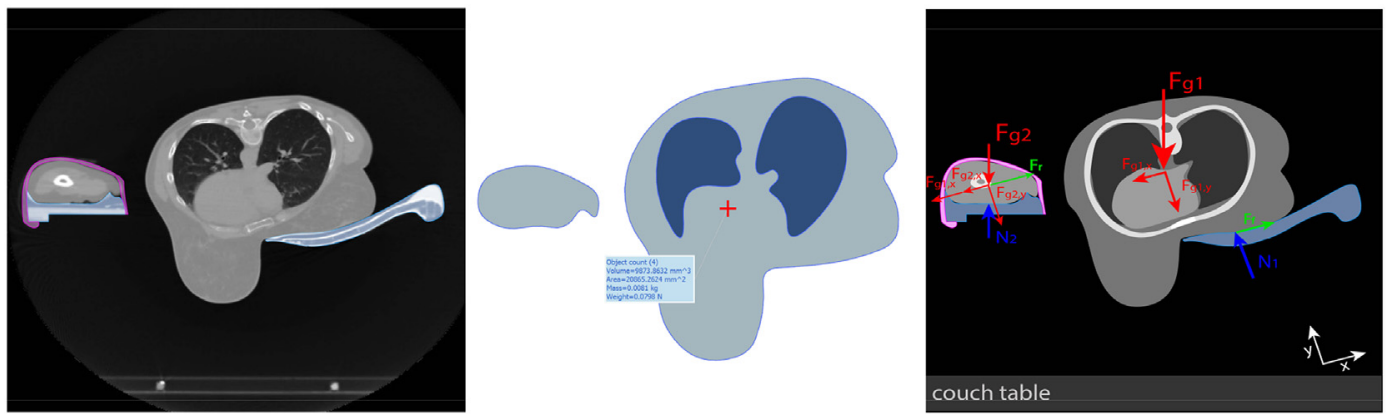

Fig. 1. Process of the simplified patient FBD and its centre of gravity (COG) determination: Left: transverse section of CT-scanned patient with contoured patient support device and fixation strap. Middle: CAD-simulation of COG (red cross) with dark blue area as lung region $\left(0.3 \mathrm{~g} / \mathrm{cm}^{3}\right)$ and light blue area for rest of the body $\left(1 \mathrm{~g} / \mathrm{cm}^{3}\right)$. Right: derived CT-illustration with simplified FBD. Blue areas represent the patient support device, purple area represents the arm fixation strap. Red vectors represent gravity forces, blue vectors represent normal forces and green vectors represent reaction and friction forces. (For interpretation of the references to colour in this figure legend, the reader is referred to the Web version of this article.)

affect patient discomfort: wrong anatomical proportions, partially body support and uneven pressure distribution (Cordell et al., 1995).

In the case of MDD we could say feelings of discomfort can be associated with physical factors while comfort can be associated with feelings of relaxation and well-being (Zhang et al., 1996). Some additional factors which can influence comfort and discomfort for patients using or lying on medical devices are: treatment time, fixation and patient immobilization, patient mobility, material properties and the design of the medical device itself.

This paper focuses on the design of the medical device itself and the relation between uncompensated internal forces in the patient freebody diagram (FBD) and discomfort.

\subsection{Breast board}

Devices used for this study are so called breast boards (Fig. 3). A breast board is a patient support device for breast (and lymph node) radiotherapy. Prone position breast radiotherapy has several advantages in comparison with supine position (Monten et al., 2015; Mulliez et al., 2015; Soegaard and Dam, 2013). However, it is challenging for patients requiring whole breast irradiation (WBI) and lymph node irradiation (LNI). Our aim was to investigate a new, comfortable patient position and device which is suited for both WBI and LNI.

\subsection{Objective}

The objectives of this study were: (1) to specify pain- and pressure points on the currently used breast board ( $\mathrm{AIO}^{\mathrm{TM}}$ Orfit) and compare
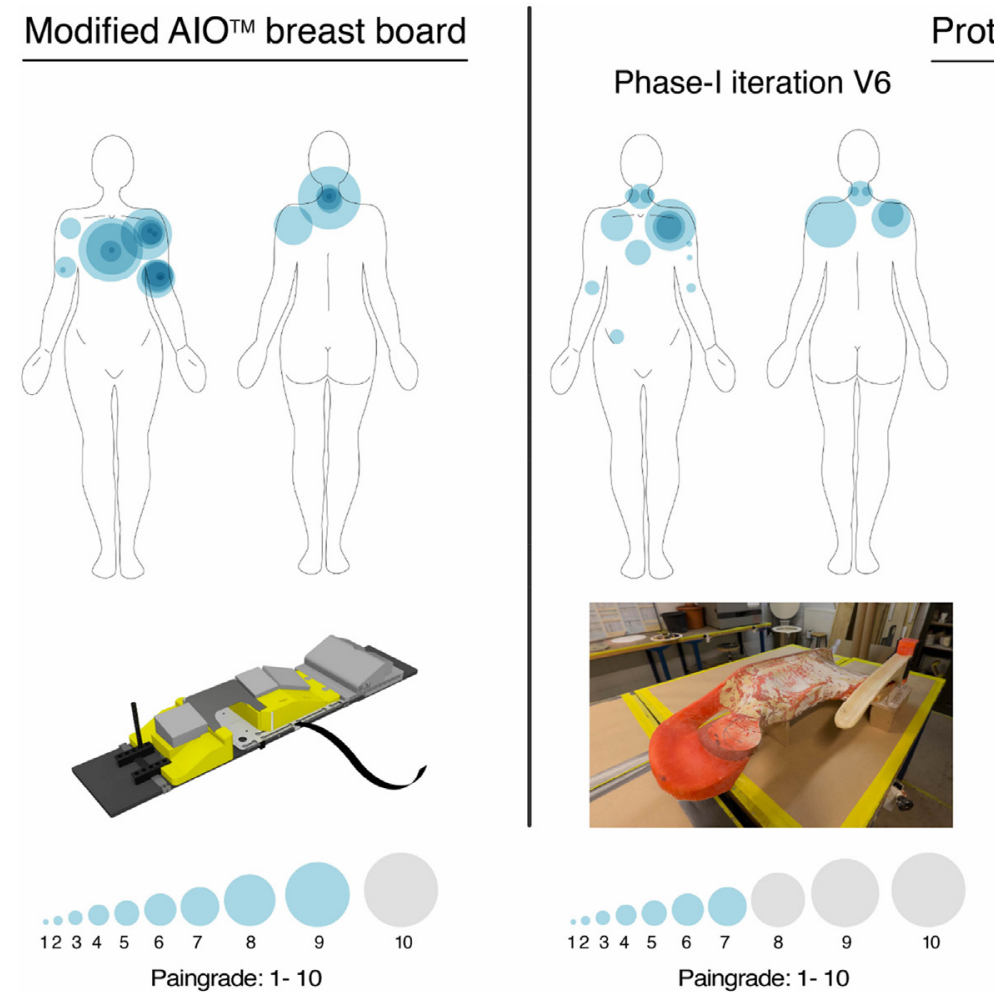

\section{Prototypes}

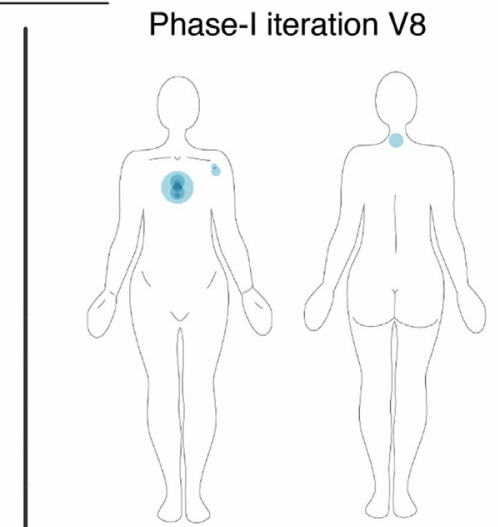

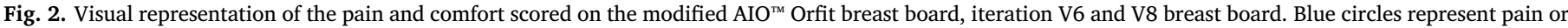

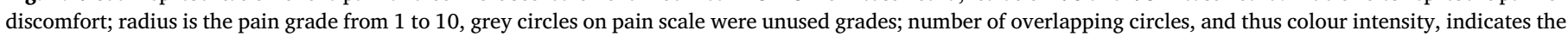

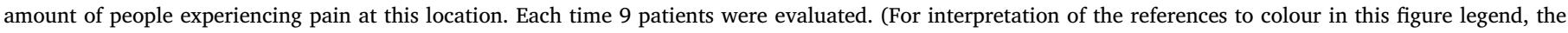
reader is referred to the Web version of this article.) 

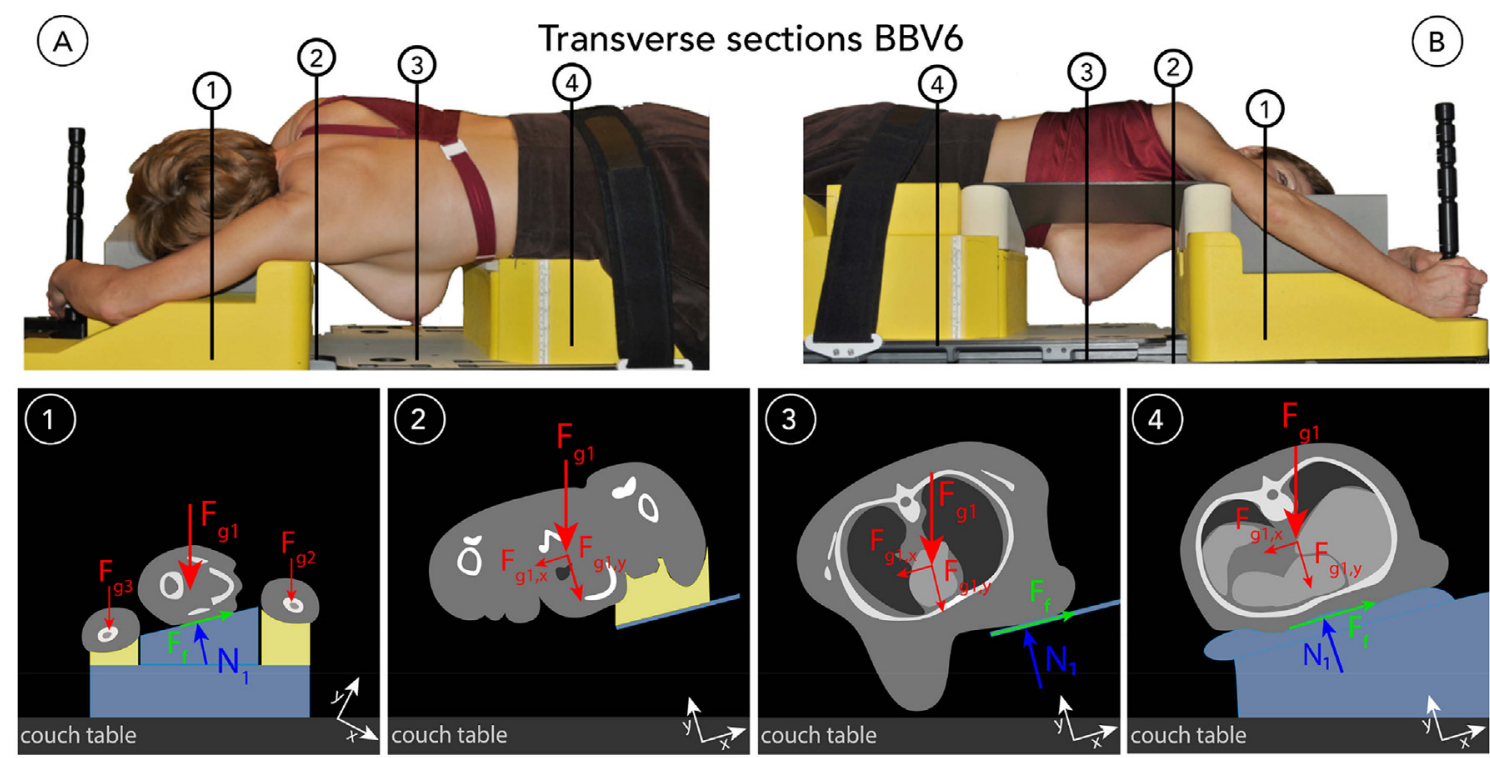

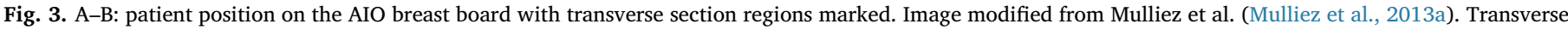

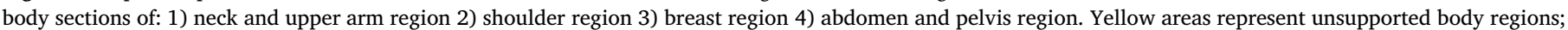

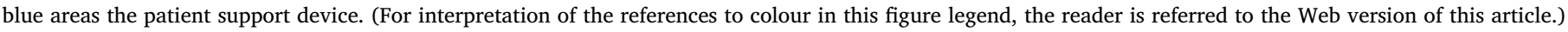

them with the patient FBD, to find a relation between discomfort and uncompensated forces; and (2) to verify a force neutral patient FBD reduces discomfort and possible pain or pressure points.

\section{Materials \& methods}

\subsection{Design approach}

During this research project, we used the research through design method (Soegaard and Dam, 2013) and a design thinking approach (Dorst, 2011). Prototype development was done through iterative prototyping. In this manuscript we explain our used workflow of patient position optimization through FBD analysis, CT analysis and pain \& comfort evaluation. Evaluated parameters were patient position (anatomy), comfort assessment and beam accessibility.

\subsection{Participants}

Nine female volunteers [ex-patients (who had been treated previously in prone position) and staff] with wide anatomical variation were selected [weight: $48-100 \mathrm{~kg}$; length: 146-184 cm; Breast size: small to large (not recorded)]. They all participated during every user test, treatment position optimization, and pain and comfort evaluation. All volunteers were familiar with the $\mathrm{AIO}^{\mathrm{TM}}$ breast board.

\subsection{Apparatus}

The device used for the first part of the study was a modified prone breast board (AIO ${ }^{\mathrm{TM}}$ Orfit) for WBI. This device has already proven to be superior than the supine device and is used as a standard treatment device at our centre (Monten et al., 2015; Soegaard and Dam, 2013; Dorst, 2011; Veldeman et al., 2010). This device is a tabletop model, which is elevated above the table so the breast can hang freely through the device. Favourable beam access is often obstructed by the design of the device, patient anatomy and table.

Prototypes used for the second part of the study were prone breast boards suitable for WBI + LNI. Fundamental parameters of the prototype were derived from the modified $\mathrm{AIO}^{\mathrm{TM}}$ breast board (Mulliez et al., 2013a). The two major iterations (Version V6 and V8) were used for the volunteer study. Previous iterations were used for establishment of the prone crawl position and local improvements such as arm-, breast-, hipand head support. For each region, patient position and shape of the breast board was analysed and redesigned in order to reduce discomfort reported by volunteers and counter-act suspected uncompensated forces leading to instability and internal body strain. The wedge, which supports the contralateral breast (i.e. non-treated breast), was redesigned for better patient comfort and reduction of imaging artefacts.

\subsection{Procedure}

Each breast board device (AIO ${ }^{\mathrm{TM}}$ and prototypes) was placed on top of the CT-simulator couch blade.

Volunteers on the $\mathrm{AIO}^{\mathrm{TM}}$ breastboard were positioned in prone position with both arms elevated. Two handles were installed above the head for a better stabilization. A slight roll of 15 degrees of the torso ensured better treatment accessibility. Volunteers on the prototypes were positioned in prone position and with the arm at the treated side (ipsilateral) alongside the body. Ipsilateral shoulder and chest was unsupported, resulting in the possibility for regional lymph node irradiation. The arm at the opposite side was positioned above the head resembling a phase of a crawl swimming movement. The whole patient was tilted with a roll of $15^{\circ}$ i.e. treated side of the patient is positioned lower. The ipsilateral breast is hanging through the device which is suspended over the table, resulting in excellent radiotherapeutic anatomy and beam access for WBI + LNI (Mulliez et al., 2013b; Deseyne et al., 2017). Volunteers were asked to lie immobile for at least $10 \mathrm{~min}$.

After each session, volunteers were asked to fill in a survey considering pain and comfort evaluation.

Six regions could be rated: neck, right shoulder, left shoulder, thorax, right arm, left arm. A numeric pain rating scale (NRS) from 0 to 10 going from no pain to an unbearable pain experienced respectively 
was used. NRSs are considered to be the most versatile and commonly used scales for pain intensity assessments (Hjermstad et al., 2011). The evaluation form is added as appendix.

\subsection{Data analysis}

Transverse CT-images of female patients (positioned on $\mathrm{AIO}^{\mathrm{TM}}$, V6 and V8 breast board prototype), spaced with approximately $5 \mathrm{~mm}$ in cranio-caudal direction, were used for data analysis. Additional CTimages of female Thiel-embalmed cadavers were used for further device improvements. Derived from patient CT-images, simplified CT-illustrations were sketched (Fig. 1-right) and centre of gravity (COG) was defined using CAD simulation software. A mass density of $0,3 \mathrm{~g} / \mathrm{cm}^{3}$ and $1 \mathrm{~g} / \mathrm{cm}^{3}$ was used for lung and rest of the body respectively. FBD forces in complex support regions (head/upper arms; shoulders/neck; breast; abdomen/pelvis) were empirically derived from CT-images and transferred onto CT-illustrations. Interacting-, un-compensated forces and moment of forces were defined. Fig. 1 illustrates this process.

For each volunteer, pain and comfort scores were visualized on a sketch of a female body (Fig. 2). Each circle represents a painful region reported by a volunteer. Size of the circles represent pain scores going from 1 as smallest circle, to 10 as largest circle. The colour intensity represents multiple pain reports in the same region.

Results of pain and comfort evaluation from the first study were analysed and compared with their FBD section. Subsequently, this data was used for the next prototype iteration to optimize patient position and comfort and acquire a force-neutral FBD in every body region. Data from prototype iteration V6 was used for the adjustments made on next prototypes. Data from prototype iteration V8 was analysed and used for comparison between a comfortable patient position and inner forceneutral patient FBD.

\section{Results}

\subsection{Modified $A I O^{\mathrm{TM}}$ Orfit breast board}

\subsubsection{Pain and comfort analysis}

High discomfort was reported in both upper arms and axilla (Fig. 2left), especially on the treated breast side (Fig. 3A). The arm support did not evenly support the arm and caused pressure points (Fig. 3-top, region between section 1 and 2). Soft napkins were often placed on the arm supports to distribute pressure. Pain at the antero-medial side of the ipsilateral upper arm was caused by arm elevation. Volunteers had to apply a counter-force with the same arm to maintain a stable position (handgrip visible on Fig. 3A). Pain was frequently reported at the neck region, caused by an uncomfortable head-position and support. Repeatedly pain was reported at the sternum near the medial edge of the wedge, supporting the contralateral breast. An uncomfortable feeling of rolling off the device and being in an unstable position was often reported since no lateral side support for torso, hip or leg was available on the device.

\subsubsection{FBD upper arms, shoulders and neck region}

Poor arm support surface (Fig. 3-top) results in partial support of upper arms and shoulders (Fig. 3.1). Fig. 3.2 displays the unsupported contralateral axilla. Most of the forces are thus loaded on the ipsilateral axilla- and arm support. The hard edge of the foam wedge results in a concentrated load on the upper arm. Since no lateral support is available, patients tend to slide off the device. This was partially compensated by holding the handlebars, which results in a stressed arm position. Neck strain and an uncomfortable head position was often reported.

\subsubsection{FBD breast region}

The FBD at the breast region (Fig. 3.3) consists of following forces: partial gravity force of the patient weight $\left(\mathrm{F}_{\mathrm{g} 1}\right)$, normal force $\left(\mathrm{N}_{1}\right)$ perpendicular to the support surface, and friction force $\left(\mathrm{F}_{\mathrm{f}}\right)$ between wedge and breast. $F_{f}$ is rather small since the wedge has a smooth surface and the contralateral breast is supported with a soft fabric unilateral breast holder (Van de Velde, Schellebelle, Belgium) used to retract the contralateral breast away from the treated breast. The $y$ component $\left(\mathrm{F}_{\mathrm{g} 1, \mathrm{y}}\right)$ is compensated by $\mathrm{N}_{1}$. The $\mathrm{x}$-component $\left(\mathrm{F}_{\mathrm{g} 1, \mathrm{x}}\right)$ is marginally compensated by the $\mathrm{F}_{\mathrm{f}}$. Since the COG is located left from the wedge and no lateral support is present, a constant moment of force occurs and tends to roll the patient downwards off the device. This moment of force is compensated in other body regions such as shoulderneck region or abdomen-pelvis region.

\subsubsection{FBD abdomen and pelvis region}

The device supports the whole abdomen region (Fig. 3.4). $F_{g 1, y}$ is fully compensated by $\mathrm{N}_{1} . \mathrm{F}_{\mathrm{g} 1, \mathrm{x}}$ is partially compensated by $\mathrm{F}_{\mathrm{f}}$. A constant moment of force occurs and tends to roll the patient downwards off the device. This moment of force, together with the uncompensated moment from the breast region, causes internal body torque and needs to be partially compensated with pelvis and legs; resulting in a constant internal body tension and consequently stressed position. The belt for hip fixation cannot be properly fastened and provides inadequate support since latero-lateral movement is still possible.

\subsection{Breast board prototype V6}

\subsubsection{Development}

The sixth prototype iteration (V6) was developed for the establishment of a suitable prone crawl patient position; different arm- and head positions were tested. The prototype has an upward contralateral-axilla support, which evenly supports the shoulder region (Fig. 4B). The head is positioned "face down". Ipsilateral arm is supported next to the body for a natural position of shoulder and lymph node region, resulting in favourable access for LNI (Fig. 4A). The arm support also acts as a lateral support for the upper body. The concave shaped upper surface of the wedge supports the contralateral breast (Fig. 4.3). The medial side of the wedge is rounded for a better pressure distribution at the sternum. An adjustable hip support module is installed and serves as a lateral support for abdomen and pelvis region. The arm support is mounted onto the hip support.

\subsubsection{Pain and comfort analysis}

As predicted from previous iteration tests, prototype iteration V6 had an overall improvement of pain and comfort scoring in comparison with the AIO breast board (Fig. 2-middle). Uneven support of the contralateral arm resulted in some discomfort. Due to partial axilla support, moderate pain was reported. The ipsilateral arm support scored better on pain and comfort evaluation in comparison with the $\mathrm{AIO}^{\mathrm{TM}}$ breastboard. Pain at ipsilateral shoulder was reported since the cranial part of the arm support did not evenly support the shoulder. Sternal pressure was reduced by the ipsilateral arm- and shoulder support and the concave shaped wedge resulted in better support of the contralateral breast. No pain or discomfort was reported in abdomen and pelvis region. Moderate pain was reported in neck region due to a too high head support.

\subsubsection{FBD contralateral arm, shoulders and neck region}

In the shoulder region, $F_{g 1, y}$ fully compensated by $N_{1}$ (Fig. 4.2). $F_{g 1, x}$ is partially compensated by $\mathrm{F}_{\mathrm{f}}$. Since the COG is located left from the shoulder support and no lateral support is present, a constant moment 


\section{Transverse sections BBV6}
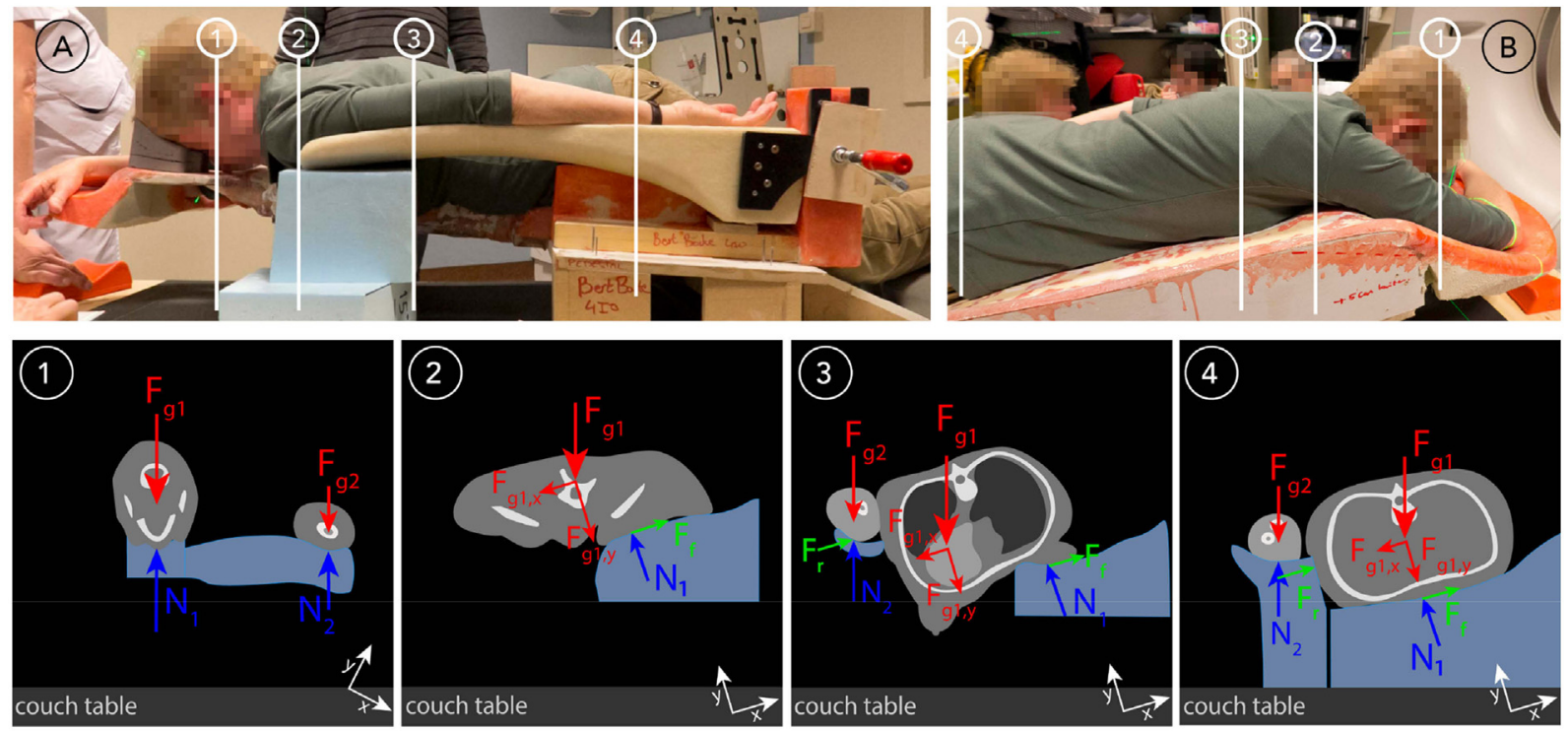

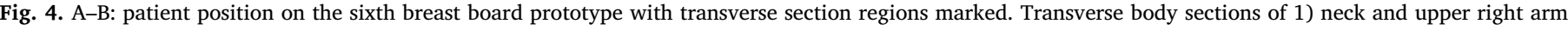

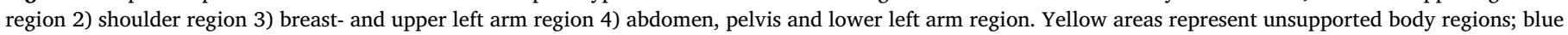
areas the patient support device. (For interpretation of the references to colour in this figure legend, the reader is referred to the Web version of this article.)

of force occurs. This is partially compensated by the weight of the contralateral arm (Fig. 4.1) and new position of the ipsilateral arm (Fig. 4.3). Some stress and torsion were reported in the neck due to uneven contralateral arm- and axilla support. The arm could not be properly positioned and thus immobilized, resulting in bigger pressure load on ipsilateral arm and shoulder. The hard surface of the arm support resulted in a concentrated load.

\subsubsection{FBD breast region}

The moment of force which caused rolling off the device is fully compensated by the arm support at the ipsilateral side. Upper body weight is now divided over $\mathrm{F}_{\mathrm{g} 1}$ and $\mathrm{F}_{\mathrm{g} 2} . \mathrm{F}_{\mathrm{g} 2}$ consists of the arm weight and partial upper body weight. $\mathrm{F}_{\mathrm{g} 1, \mathrm{x}}$ is compensated by $\mathrm{F}_{\mathrm{f}}$ and $\mathrm{F}_{\mathrm{r}} . \mathrm{F}_{\mathrm{g} 1, \mathrm{y}}$ is compensated by $\mathrm{N}_{1}$.

\subsubsection{FBD abdomen and pelvis region}

The moment of force at the pelvis region, which caused the patient to roll off the device, is fully compensated by the lateral hip support. $\mathrm{F}_{\mathrm{g} 1 \mathrm{x}}$ is fully compensated by $\mathrm{F}_{\mathrm{r}}$. The smooth surface of the breast board results in minor friction between patient and device. This is advantageous for patient re-positioning.

\subsection{Breast board prototype $V 8$}

\subsubsection{Development}

Pain and comfort analysis, beam access range and 3D-anatomy of patient CT-images, positioned on the sixth prototype, were evaluated for the development of prototype iteration V8. Transverse CT-images of cadavers were used for additionally device improvement and redrawing of the patient support surface to human anatomy (Fig. 5). Transverse CT-slices were edited in a CAD-CAM environment. In-between the edited slices, surface shapes were interpolated. Upper surface of the prototype was re-drawn on the transverse images according to knowledge gained during previous prototype iterations and patient CTscans.
Green area in Fig. 5 represents transverse sections of prototype iteration V6. Yellow area represents the new suggested patient support surface. A new upper contralateral arm support (yellow part Fig. 5.2) was redesigned to an inverted wing (i.e. surface cranking downwards in lateral and cranial directions). This evenly supports contralateral axilla and arm and forces the shoulder to slide over the axilla support (Fig. 5.1). This design counteracts the tendency of rolling off the device and thus provides a more stable position by counteracting lateral and downward movements.

By reshaping the ipsilateral arm support into a flat surface, the arm could be more freely positioned by flexing the elbow. The upper body partially rests onto the ipsilateral arm support alongside the body. This support can be both anterior-posterior and latero-lateral positioned for adjusting patient roll and adapting to different body types. The arm-hip module provides support for abdomen and pelvis region. This design physically eliminates the possibility for patients to roll off the device.

\subsubsection{Pain and comfort analysis}

No pain and discomfort were reported for contralateral arm and shoulder (Fig. 2-right). Contralateral axilla and upper arm was more evenly supported. The shoulder, sliding over the axilla support, was reported to be comfortable. Comfort of the ipsilateral arm was improved. Minor pain was reported at the sternum. This sternal pressure can be related to hard support surface and an inadequate support of the contralateral breast.

\subsubsection{FBD contralateral arm, shoulders and neck region}

$\mathrm{F}_{\mathrm{g} 2 \mathrm{x}}$ acts as a downwards-right force which causes the contralateral arm to slide downwards over the axilla support, resulting in a laterolateral immobilization of the shoulder region (Fig. 6.2). The head is positioned downwards, resulting in minimized stress in neck region (Fig. 6.1). Although no pain was reported at the contralateral axilla and arm, the yellow area in Fig. 6.2 indicates that the axilla was not fully supported. The force $\mathrm{F}_{\mathrm{g} 1}$ in shoulder region needs to be compensated by arms, neck or abdomen region. In the long term, this could result in 


\section{1: transverse section (shoulder)}
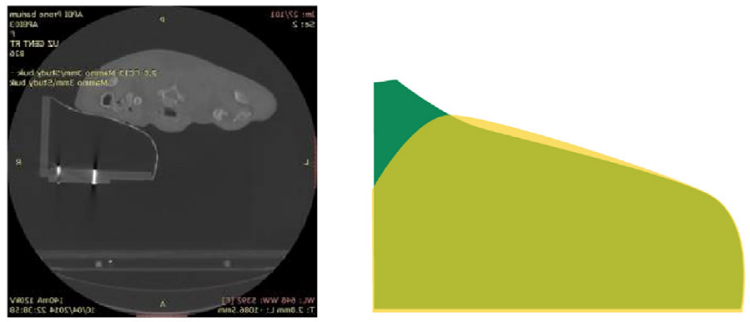

\section{3: transverse section (elbow)}

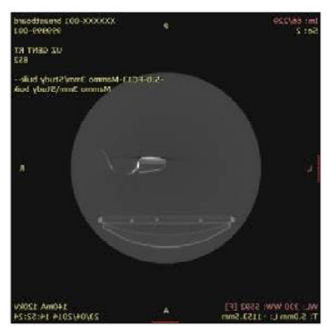

\section{2: transverse section (upper arm)}
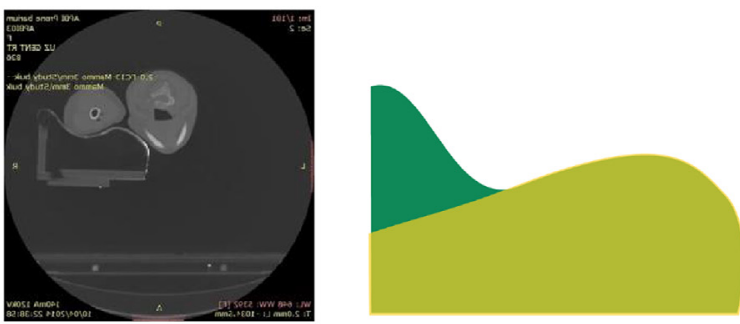

\section{4: transverse section (lower arm)}

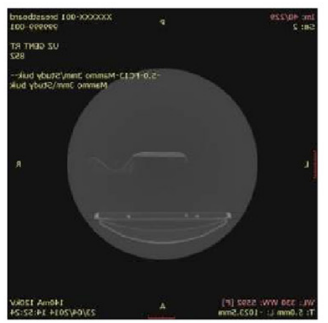

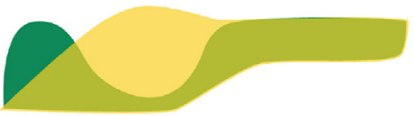

Fig. 5. Transverse sections of CT-scanned cadavers on prototype iteration V6. Green: representation of prototype V6 section silhouette. Yellow: suggested new support area for axilla and contralateral arm. (For interpretation of the references to colour in this figure legend, the reader is referred to the Web version of this article.)

strain or pressure points.

\subsubsection{FBD breast region}

Y-component $\left(\mathrm{F}_{\mathrm{g} 1, \mathrm{y}}\right)$ of the gravity force in Fig. 6.2 is compensated by $\mathrm{N}_{1}$ since the concave curved wedge more evenly distributes pressure. $\mathrm{X}$-component $\left(\mathrm{F}_{\mathrm{g} 1, \mathrm{x}}\right)$ is marginally compensated by friction force $\mathrm{F}_{\mathrm{f}}$.

The main compensation of $\mathrm{F}_{\mathrm{g} 1, \mathrm{x}}$ is caused by $\mathrm{F}_{\mathrm{r}}$ of the arm support. $F_{r}$ eliminates the possibility of rolling off the device, caused by the moment of force. $\mathrm{N}_{2}$ of the arm support in Fig. 6.3 compensates $\mathrm{F}_{\mathrm{g} 2}$, which is composed by the weight of the contralateral arm and partial body weight (transferred from shoulder region).

\subsubsection{FBD abdomen and pelvis region}

The lateral hip support fully immobilizes abdomen and pelvis region. Reaction force Fr fully compensates possible roll-off caused by $\mathrm{Fg}_{1, \mathrm{x}}$ (Fig. 6.4).

\section{Transverse sections BBV8}
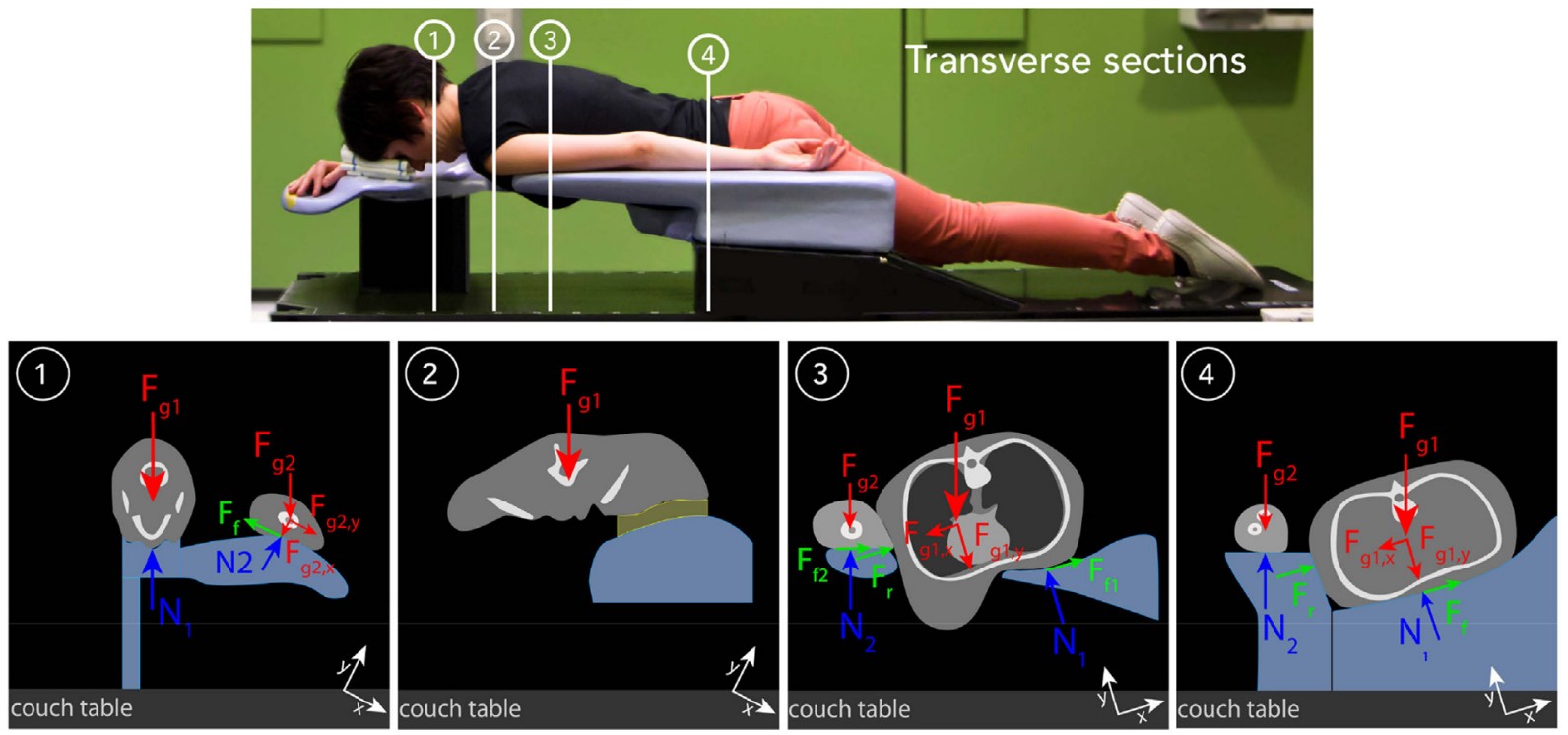

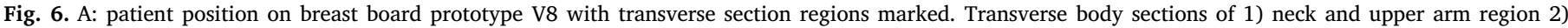

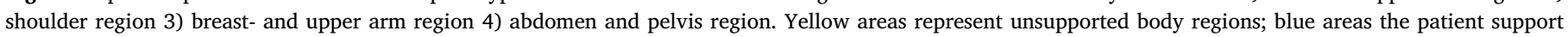
device. (For interpretation of the references to colour in this figure legend, the reader is referred to the Web version of this article.) 


\section{Discussion}

After searching separate keywords such as: discomfort, user comfort, medical devices, FBD, identifying factors, patient immobilization, radiotherapy, postural loading, revalidation, etc. only published research was found concerning discomfort and postural loading at the joints (Boussenna et al., 1982), identifying factors of comfort using hand tools (Kuijt-Evers et al., 2004; Kong et al., 2012) identifying factors of comfort and discomfort in sitting (De Looze et al., 2003; Cordell et al., 1995), postural load (Chung et al., 2005; Vergara and Page, 2000) and muscle fatigue during truck driving (Wilder et al., 1994). No directly relevant published research was found on the relation between discomfort and uncompensated internal forces affecting patient comfort and medical performance. Especially for radiotherapy devices no data was found. Therefore, the aim of this study was to find a relation between uncomfortable patient positions (on medical devices) and the patient's FBD. One of the possible reasons why there is not much published research about MDD is the understandable reluctance of companies to disclose commercially sensitive information about the development process (Martin et al., 2012).

\subsection{Success of the study}

The first part of the study analysed the relation between the FBD and discomfort on the modified AIO breast board. FBD analysis demonstrates that uncompensated forces are related to pain and comfort scores. Pain reported in the neck area can be related to an opposite rotation $\left(80^{\circ}-90^{\circ}\right)$ of the cervical vertebrae and upper thoracic vertebrae. This head rotation is rather extreme since mean axial cervical range of motion for female persons between the age of 50 and 65 is $140,8^{\circ}$ (SD of $18,4^{\circ}, \mathrm{n}=60$ ) (Roy et al., 1996). Shoulders and upper arms need to compensate the unstable upper body position to prevent it from rolling off the device, which results in muscle contraction of arms and firmly gripping the handholds to maintain a stable position. Pain reported at the sternum can be related to the whole weight of the torso resting on the wedge. The flat wedge surface unevenly supports the breast and consequently causes a pressure peak at the medial edge. Since the torso is not supported on the ipsilateral side, other sections such as shoulders and hips need to compensate this roll effect, resulting in strain and torque.

Prototype V6 was the establishment of a prone crawl position prototype which counter-acted most moments of force and uncompensated forces. The ipsilateral arm support counteracts the moment of rolling off the device and serves as a lateral support for the torso. The hip support (caudal part of the arm support) immobilizes abdomen and pelvis region. Although every internal body force was compensated, discomfort was still reported. Sub-optimal support surface areas resulted in partial body support and as De Looze et al. (De Looze et al., 2003) states, uneven pressure distribution resulted in discomfort and local pain points.

With prototype iteration V8, we were able to counter-act all uncompensated forces. Except for minimal pain reported at sternum and ipsilateral arm, all pain points were eliminated. This was done by support surface optimization: better pressure distribution, improved support surface contact, local foam sheet application and adjustable support modules. Most people reported this iteration as comfortable.

By Thiel soft-fix embalming, the skin and muscles remain flexible and allow the limbs to be moved in a natural way (Thiel, 1992), (De Crop et al., 2012). In addition, no internal body- or muscle strain is possible. This was favourable for replicating a "relaxed" a "natural" body position. When analysing CT-images of cadavers, we noticed that they were very similar to patient CT-images. Subsequently these images could be used for additional position analysis.

By analysing CT-images of specific body regions of each time one subject with average body proportions, we were able to derive a general simplified FBD. Uncompensated forces could be specified and directly related to pain and comfort analyses of every volunteer. Based on transverse CT-image- and FBD analysis, we were able to improve the prototype design and reduce overall discomfort. The results demonstrate that further prototype iterations could be executed with a general FBD analysis of one subject. A similar method approach could be advantageous in other research projects.

\subsection{Need for a user centred approach and iterative prototyping}

Many authors have stated that the application of a user centred design approach in MDD has added value, and provides guidance on the theory behind it (Thiel, 1992; De Crop et al., 2012). Although there are a number of possible reasons why designers wait with consulting of the users. As Martin et al. (2010) states: "Currently, users are generally not brought into the developmental process until after the design brief for a new product has been produced.

This may be because medical devices are frequently technology driven rather than resulting from an identified un-met need". Two other notable reasons are time and financial constraints (Martin et al., 2012).

During our iteration phases, time and money was conserved by testing every prototype with volunteers on a small scale and directly evaluating the results with medical researchers and nurses. With this iterative method, fast proof of concepts for sub-problems were produced with a minimal effort of time and money: the eighth prototype iteration combined every pervious modification and fused this into a functional prototype, which offered a comfortable patient position and could be used as a reference prototype for further MDD and research (Veldeman et al., 2010; Mulliez et al., 2013b).

To enable incorporation of new features to prototypes with greater ease and lower cost, identification of user needs and co-operation of the user is important for the development process of new medical products. As Martin et al. (2010) states, if the users were not brought into the developmental process in an early stage, the devices would probably be medical functional but would not meet the desired patients comfort. A good example of a medical device with a user centred design approach and a multidisciplinary team is the development of a laparoscopic instrument (Loring and Lemieux, 2010).

\subsection{Study limitations}

This study was conducted with 9 volunteers for pain and comfort evaluation, each time one patient was CT-scanned on the AIO, V6 and V8 device for the establishment of the FBD's. Since ethics committee allowed us to only scan one patient per prototype, additional cadavers were used for extra CT-scanning. These scans were used for internal body anatomy analysis and prototype optimization.

Bigger sample sizes (particularly for CT-imaging) could be advantageous but are harder to obtain ethics approval, especially early during the development phase of medical devices. In addition, this could be counter-productive and unpractical since first prototype iterations evolved rapidly. During further iterations (with more advanced prototypes), bigger sample sizes were used (Boute et al., 2017).

Since patients were only positioned for approximately $10 \mathrm{~min}$ (instead of $15 \mathrm{~min}$ for a real treatment), a comparative study was performed where ten patients received half of their WBI treatment sessions on the crawl breast board prototype and the other half on $\mathrm{AIO}^{\mathrm{TM}}$ device 
(Boute et al., 2017). In general, the prone crawl device scored best.

\subsection{Immobilization}

Positioning and immobilization of patients is extremely important during radiation therapy (Rosenthal et al., 1993). The sole focus of a fixation device is to provide each time reproducible patient positioning throughout the duration of patient treatment sessions. Immobilization can be defined as the act of limiting movement through fixation of a body part in order to facilitate treatment, and thus, cure the disease (Mullaney et al., 2012). When a patient is not properly immobilized, or not positioned in a natural and reproducible position, the patient can be at risk of having a reduced cure probability due to complete or partially missing of the target volume. Also increased accidental dosing of sensitive organs can result in adverse side effects (Mullaney et al., 2012).

\subsection{Other factors of discomfort}

The time when a patient lies on the device or is immobilized during treatment, can influence discomfort (Lerner and Moscati, 2000). A patient could experience no pain or discomfort in the first radiotherapy session while after several sessions the patient could experience severe pain, discomfort and in worst case the treatment could be interrupted. Patient immobilization done by fixation can result in pain or discomfort caused by hard support areas, tensioned belts, local pressure areas, etc. ... (Mulliez et al., 2013a; Grocott et al., 2007; Rosenthal et al., 1993). Patient mobility: Some elder patients have painful joints and mobility issues caused by arthrosis, arthritis or other conditions (Petersson, 1986). Additionally, some patients underwent breast surgery or lumpectomy (lymph node removal), which can result in restricted movement possibilities.

Material properties can be related to both comfort and discomfort. A surface can have a high or low friction finishing, anti-slip, soft/hard (Lerner et al., 1996).

\subsection{Next iterations}

The head and neck support were still an issue. Frontal support resulted in a rather unstable head position, and in some cases, neck pain. A head position with in-line orientation of thoracic- and cervical vertebrae could be more appropriate. In several cases the inverted wing support of breast board prototype V8 did not fully support the patient contralateral axilla and upper arm. This is solved in prototype V9 and V10. They were developed by the same method of previous iterations and used for arm-, hip- and head support optimization. The general patient position was not changed. V9 was used for a pilot study which compared five patient treatment simulations in supine and prone crawl position. This study demonstrates that the patients, positioned on the prone crawl device, have favourable properties of sparing vital organs and good breast and nodal target coverage compared to the supine position (Deseyne et al., 2017).

Additionally, a comparative study $(\mathrm{n}=10)$ was performed with the AIO breast board and prototype V10. This study confirms that the prone crawl patient position results in better comfort, stability, and set-up precision and permits a vast range of new favourable beam directions for WBI + LNI in comparison with the commonly used prone position (Boute et al., 2017).

The last iteration is used for a case study with bigger sample size $(n=40)$ and real patient treatments. Patients are evaluated after the first- and last treatment session (15 sessions of proximally each $15 \mathrm{~min}$ ).

\section{Conclusion}

By FBD analysis of patients and cadavers through CT-images, it was possible to identify uncompensated internal body forces, compensate them and achieve a force neutral patient position. This resulted in good patient positioning and eliminated discomfort. This workflow could be potentially beneficial for the development of other medical devices.

\section{Conflicts of interest}

Ghent University owns the patent application entitled Radiotherapy Board and Couch [WO2015144654A1] filed on 25.03.2014 for which Wilfried De Neve, Bruno Speleers, Bert Boute and Liv Veldeman own the intellectual rights.

\section{Acknowledgements}

This work was financed through Cancer Plan Action 29 by the Federal Public Service of Health, Food Chain Safety and Environment, Belgium (KPC_29_008) and by a Career Catalyst Grant from Susan G. Komen (Grant 377841). Prototype research is funded by StarTT 241 grant of the Industrial Research Fund, Ghent University thanks to management by Lieve Nuytinck, Bimetra, Clinical Research Centre, Ghent University.

Anatomy experiments were funded by Foundation against Cancer, grant 2012-200. Annick Van Greveling is funded by a grant of ThinkPink. Bert Boute is recipient of a research grant Emmanuel van der Schueren of Kom op tegen Kanker, Liv Veldeman is funded by a grant of Kom op tegen Kanker. 
Appendix A. Pain and comfort evaluation form for the AIO breast board.

\begin{tabular}{c}
$\substack{\text { UNIIIIII } \\
\text { GERITEIT }}$ \\
\hline
\end{tabular}

\section{Breast board evaluation form}

Patiënt nr:

Length:

weight:

Please fill in your pain experience after lying on the device for at least 10 minutes. Mark with an $X$

\begin{tabular}{|c|c|c|c|c|c|c|c|c|c|c|c|}
\hline & No & \multicolumn{10}{|c|}{ yes, $(1=$ few; $10=$ unbearable pain $)$} \\
\hline & 0 & 1 & 2 & 3 & 4 & 5 & 6 & 7 & 8 & 9 & 10 \\
\hline Did you experience pain or discomfort in the neck? & & & & & & & & & & & \\
\hline $\begin{array}{l}\text { Did you experience pain or discomfort in the right } \\
\text { shoulder? }\end{array}$ & & & & & & & & & & & \\
\hline Did you experience pain or discomfort in the left shoulder? & & & & & & & & & & & \\
\hline Did you experience pain or discomfort in the right arm? & & & & & & & & & & & \\
\hline Did you experience pain or discomfort in the left arm? & & & & & & & & & & & \\
\hline Did you experience pain or discomfort in the thorax? & & & & & & & & & & & \\
\hline Did you have the feeling of rolling off the table? & & & & & & & & & & & \\
\hline
\end{tabular}

Where did you experience pain (mark with $\mathrm{X}$ ) or discomfort (mark with $\mathrm{O}$ ).

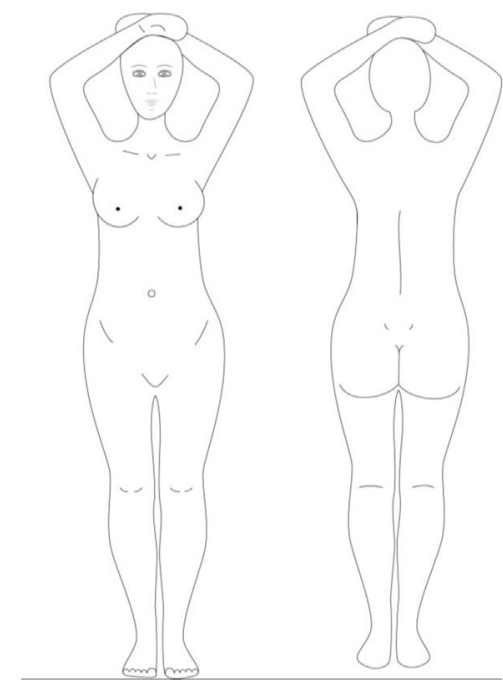

Remarks:

\section{References}

Balka, E., Doyle-waters, M., Lecznarowicz, D., Fitzgerald, J.M., 2006. Technology, governance and patient safety : systems issues in technology and patient safety. Int. J. Med. Inf. 35-47.

Boussenna, M., Corlett, E.N., Pheasant, S.T., 1982. The relation between discomfort and postural loading at the joints. Ergonomics 25 (4), 315-322.

Boute, B., De Neve, W., Speleers, B., Van Greveling, A., Monten, C., Van Hoof, T., Van de Velde, J., Paelinck, L., De Gersem, W., Vercauteren, T., Detand, J., Veldeman, L., Jul. 2017. Potential benefits of crawl position for prone radiation therapy in breast cancer. J. Appl. Clin. Med. Phys. 18 (4), 200-205.

Chung, M.K., Lee, I., Kee, D., 2005. Quantitative postural load assessment for whole body manual tasks based on perceived discomfort. Ergonomics 48 (5), 492-505.

Clarkson, P.J., Buckle, P., Oleman, R.C., Stubbs, D., Ward, J., Lane, R., Nd, J.B.O.U., 2004. Design for Patient Safety: a Review of the Effectiveness of Design in the UK Health Service.

Cordell, W.H., Hollingsworth, J.C., Olinger, M.L., Stroman, S.J., Nelson, D.R., 1995. Pain and tissue-interface pressures during. Ann. Emerg. Med. 20 (July), 31-36.

De Crop, A., Bacher, K., Van Hoof, T., V Smeets, P., Smet, B.S., Vergauwen, M., Kiendys, U., Duyck, P., Verstraete, K., D'Herde, K., Thierens, H., 2012. Correlation of contrastdetail analysis and clinical image quality assessment in chest radiography with a human cadaver study. Radiology 262 (1), 298-304.

De Looze, M., Kuijt-evers, L., Van dieën, J., 2003. Sitting comfort and discomfort and the relationships with objective measures. Ergonomics 46 (August 2014), 37-41.

Deseyne, P., Speleers, B., De Neve, W., Boute, B., Paelinck, L., Van Hoof, T., Van De Velde, J., Van Greveling, A., Monten, C., Post, G., Depypere, H., Veldeman, L., 2017. Whole breast and regional nodal irradiation in prone versus supine position in left sided breast cancer. Radiat. Oncol. 1-12.

Dong, H., Vanns, N., 2009. Designing an innovative pill dispenser: an undergraduate level case study of inclusive design. Des. J. 12 (1).

Dorst, K., 2011. The core of "design thinking" and its application. Des. Stud. 32 (6), $521-532$.

Edvardsson, D., Sandman, P.O., Rasmussen, B., 2006. Caring or uncaring - meanings of being in an oncology environment. J. Adv. Nurs. 55 (2), 188-197.

Edvardsson, D., Sandman, P.O., Rasmussen, B., 2008. Swedish language person-centred climate questionnaire - patient version: construction and psychometric evaluation. J. Adv. Nurs. 63 (3), 302-309.

Grocott, P., Weir, H., Ram, M.B., 2007. A model of user engagement in medical device development. Int. J. Health Care Qual. Assur. 20 (6), 484-493.

Hjermstad, M.J., Fayers, P.M., Haugen, D.F., Caraceni, A., Hanks, G.W., Loge, J.H., Fainsinger, R., Aass, N., Kaasa, S., 2011. Studies comparing numerical rating scales, verbal rating scales, and visual analogue scales for assessment of pain intensity in adults: a systematic literature review. J. Pain Symptom Manag. 41 (6), 1073-1093. 
Jensen, M.P., Chen, C., Brugger, A.M., 2002. Postsurgical pain outcome assessment. Pain 99 (1-2), 101-109.

Kong, Y.K., Kim, D.M., Lee, K.S., Jung, M.C., 2012. Comparison of comfort, discomfort, and continuum ratings of force levels and hand regions during gripping exertions. Appl. Ergon. 43 (2), 283-289.

Kuijt-Evers, L.F.M., Groenesteijn, L., De Looze, M.P., Vink, P., 2004. Identifying factors of comfort in using hand tools. Appl. Ergon. 35 (5), 453-458.

Lerner, E.B., Moscati, R., 2000. Duration of patient immobilization in the ED. Am. J. Emerg. Med. 18 (1), 28-30.

Lerner, E., Billittier, A., Moscatie, R., 1996. Effects of neutral positioning with and without padding on spinal immobilization of healthy subjects. Ann. Emerg. Med. 27, 147.

Loring, B., Lemieux, E.-A., 2010. User research improves laparoscopic instruments. Ergon. Des. Q. Hum. Factors Appl. 18 (1), 18-23.

Martin, J.L., Norris, B.J., Murphy, E., Crowe, J.A., 2010. Medical device development: the challenge for ergonomics. Appl. Ergon. 39 (3), 271-283.

Martin, J.L., Clark, D.J., Morgan, S.P., Crowe, J.A., Murphy, E., 2012. A user-centred approach to requirements elicitation in medical device development: a case study from an industry perspective. Appl. Ergon. 43 (1), 184-190.

Monten, C., Mulliez, T., Speleers, B., 2015. PD-0043: 2-year cosmetic outcome of large breasted women randomized between prone and supine whole-breast irradiation. In: 3rd ESTRO Forum, pp. 2-4 April.

Mullaney, T., Pettersson, H., Nyholm, T., Stolterman, E., 2012. Thinking beyond the cure: a case for human-centered design in cancer care. Int. J. Des. 6 (3), 27-39.

Mulliez, T., Veldeman, L., Van Greveling, A., Speleers, B., Sadeghi, S., Berwouts, D., Decoster, F., Vercauteren, T., De Gersem, W., Van Den Broecke, R., De Neve, W. 2013a. Hypofractionated whole breast irradiation for patients with large breasts: a randomized trial comparing prone and supine positions. Radiother. Oncol. 108 (2), 203-208.

Mulliez, T., Speleers, B., Madani, I., De Gersem, W., Veldeman, L., De Neve, W., 2013b. Whole breast radiotherapy in prone and supine position: is there a place for multibeam IMRT? Radiat. Oncol. 8 (1), 151.

Mulliez, T., Veldeman, L., Speleers, B., Mahjoubi, K., Remouchamps, V., Van Greveling, A., Gilsoul, M., Berwouts, D., Lievens, Y., Van den Broecke, R., De Neve, W., 2015. Heart dose reduction by prone deep inspiration breath hold in left-sided breast irradiation. Radiother. Oncol. 114 (1), 79-84.

Peters, G.A., Peters, B.J., 2007. Medical Error and Patient Safety: Human Factors in Medicine. CRC Press.
Petersson, C.J., 1986. Painful shoulders in patients with rheumatoid arthritis. Scand. J. Rheumatol. 11, 275-279.

Rosenthal, S.A., Roach, M., Goldsmith, B.J., Dogget, E.C., Picket, B., Yuo, H.S., Soffen, E.M., Stern, R.L., Ryu, J.K., 1993. Immobilization improves the reproducibility of patient positioning duting six-field conformal radiation therapy for prostate carcinoma. Int. J. Radiat. Oncol. Biol. Phys. 27 (May), 921-926.

Roy, P., Walmsley, R.P., Kimber, P., Culham, E., 1996. The effect of initial head position on active cervical axial rotation range of motion in two age populations. Spine (Phila Pa. 1976). 21 (21).

Sackett, D.L., 1997. Evidence-based medicine. Semin. Perinatol. 21 (1), 3-5.

Sackett, D.L., Rosenberg, W.M.C., Gray, J. a M., Haynes, R.B., Richardson, W.S., 1996. Evidence based medicine: what it is and what it isn't. Br. Med. J. 312 (7023), 71-72.

Sawyer, D., Aziz, K.J., Backinger, C.L., Beers, E.T., Lowery, A., Sykes, S.M., 1996. An introduction to human factors in medical devices. US Department of Health and Human Services, Public Health Service, Food and Drug Administration, Center for Devices and Radiological Health.

Scanlon, M.C., Karsh, B., Densmore, E.M., 2006. Human factors engineering and patient safety. Pediatr. Clin. north Am. 53, 1105-1119.

Soegaard, M., Dam, R.F., 2013. The Encyclopedia of Human-computer Interaction, 2nd Ed.', Encycl. Human-computer Interact, second ed. .

Tanure, R.L.Z., Echeveste, M.E.S., Zaffaroni, F.C., 2015. Assessment of health devices regarding user requirements: the emotional dimension. In: IFMBE Proceedings. vol. 49. pp. 988-991 October 2014.

Thiel, W., Jun. 1992. The preservation of the whole corpse with natural color. Ann. Anat. 174 (3), 185-195.

Veldeman, L., Speleers, B., Bakker, M., Jacobs, F., Coghe, M., De Gersem, W., Impens, A. Nechelput, S., De Wagter, C., Van Den Broecke, R., Villeirs, G., De Neve, W., 2010. Preliminary results on setup precision of prone-lateral patient positioning for whole breast irradiation. Int. J. Radiat. Oncol. Biol. Phys. 78 (1), 111-118.

Vergara, M., Page, D., 2000. System to measure the use of the backrest in sitting-posture o \$ ce tasks. Appl. Ergon. 31, 247-254.

Wilder, D., Magnusson, M.L., Fenwick, J., Pope, M., 1994. The effect of posture and seat suspension design on discomfort and back muscle fatigue during simulated truck driving. Appl. Ergon. 25 (2), 66-76.

Xiao, D.J., 2014. Ergonomic Factors during Laparoscopic Surgery Training.

Zhang, L., Helander, M.G., Drury, C.G., 1996. Identifying factors of comfort and discomfort in sitting. Hum. Factors 38 (3), 377-389. 